\title{
Pygo2 as a novel biomarker in gastric cancer for monitoring drug resistance by upregulating MDR 1
}

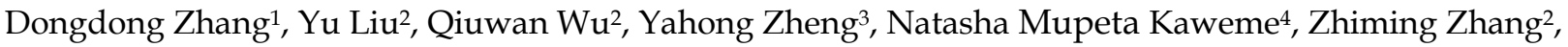 \\ Mingquan Cai ${ }^{\circledR}$ and Youhong Dong ${ }^{1 凶}$ \\ 1. Department of Oncology, Xiangyang No. 1 People's Hospital, Hubei University of Medicine, Xiangyang, Hubei 441000, China. \\ 2. Department of Medical Oncology, Xiamen Key Laboratory of Antitumor Drug Transformation Research, The First Affiliated Hospital of Xiamen University, School of \\ clinical Medicine, Fujian Medical University, Xiamen 361003, Fujian Province, P.R. China. \\ 3. Xiamen Huli District Maternal and Child Health Hospital, 361005 Xiamen, Fujian, China. \\ 4. Department of Hematology, Zhongnan Hospital, Wuhan University, Wuhan, P.R. China. \\ $\triangle$ Corresponding authors: Mingquan Cai, Department of Medical Oncology, The First Affiliated Hospital of Xiamen University, Xiamen 361003, Fujian, China. E-mail: \\ mingquan035@163.com; Youhong Dong, Department of Oncology, Xiangyang No. 1 People's Hospital, Hubei University of Medicine, Xiangyang, Hubei 441000, China. \\ E-mail: dongyouhong2005@163.com. \\ (C) The author(s). This is an open access article distributed under the terms of the Creative Commons Attribution License (https://creativecommons.org/licenses/by/4.0/). \\ See http://ivyspring.com/terms for full terms and conditions.
}

Received: 2020.09.16; Accepted: 2021.02.28; Published: 2021.03.15

\begin{abstract}
Chemotherapy is the main therapy for gastric cancer (GC) both before and after surgery, but the emergence of multidrug resistance (MDR) often leads to disease progression and recurrence. P-glycoprotein, encoded by $M D R I$, is a well-known multidrug efflux transporter involved in drug resistance development. Pygo2 overexpression has been identified in several cancers. Previous studies have shown that abnormal expression of Pygo2 is related to tumorigenesis, chemoresistance, and tumor progression. In this study, to evaluate the underlying relationship between Pygo2 and MDRI in GC, we constructed GC drug-resistant cell lines, SGC7901/cis-platinum (DDP), and collected tissue from GC patients' pre-and post-chemotherapy. We found that Pygo2 was overexpressed in GC, especially in GC drug-resistant cell lines and GC patients who underwent neoadjuvant DDP-based chemotherapy. Pygo2 overexpression may precede MDR1 and correlates with MDR 1 in GC patients. Furthermore, knock-down of Pygo2 induced downregulation of MDR1 and restored SGC7901/DDP's sensitivity to DDP. Further mechanistic analysis demonstrated that Pygo2 could modulate MDR1 transcription by binding to the MDR1 promoter region and promoting MDR1 activation. The overall findings reveal that Pygo2 may be a promising biomarker for monitoring drug resistance in GC by regulating MDR1.
\end{abstract}

Key words: Pygo2; gastric cancer; biomarker; drug resistance; MDR1

\section{Introduction}

Gastric cancer (GC) has relatively high morbidity and mortality rate around the world. Although surgery is the first choice of treatment, chemotherapy also plays an important role in treating GC as GC has a low incidence of early diagnosis. Neoadjuvant chemotherapy can diminish tumor volume, degrade tumor stage, and increase the resection rate. Postoperative chemotherapy can prevent tumor recurrence and metastasis, thus prolonging the overall survival. Palliative chemotherapy can alleviate clinical symptoms and improve the quality of life of patients. However, multidrug resistance (MDR) can eventually result in chemotherapy failure. Therefore, early detection of MDR in GC patient is essential.
Cancer cells can pump various chemotherapeutic drugs out of the cell by drug efflux transporters [1]. The occurrence of MDR is accompanied by the overexpression of multidrug efflux transporters, with the most extensively studied being multidrug resistance protein 1 (MDR1) [2]. P-glycoprotein, encoded by ATP-binding cassette subfamily B member 1 (ABCB1) or MDR1, can induce multidrug resistance by increasing drug efflux and decreasing intracellular drug concentration [3]. The overexpression of MDR1 was identified in some intrinsically drug-resistant digestive system tumors such as colorectal carcinoma, hepatocellular carcinoma, pancreatic cancer $[4,5]$, and other tumors 
[2]. Furthermore, MDR1 overexpression in breast cancer often indicated a more malignant phenotype [6] and a higher recurrence rate [7]. To date, the molecular modulation mechanism of MDR1 in gastric cancer is still unclear.

Pygopus2 (Pygo2) was initially identified as a novel functional protein of the Wingless pathway downstream in Drosophila [8]. Further studies showed Pygo 2 could regulate the $W n t / \beta$-catenin pathway via linking Pygo2 PHD domain to $\beta$-catenin [9] and activating T-cell factor (TCF)/lymphoid-enhancing factor 1 (LEF) transcription [10]. Pygo2 played an important role in transcription regulation, tissue differentiation and embryonic development [11, 12]. Pygo2 is usually maintained at a low level in normal cells but has shown an abnormally high expression level in some cancers. Overexpression of Pygo2 acted as a driver for metastatic prostate cancer and esophageal squamous cell carcinoma by enhancing tumor growth and invasion [13, 14]. Abnormal expression of Pygo2 also suggested a malignant phenotype in advanced lung cancer [15]. Moreover, Pygo2 overexpression was associated with chemoresistance and poor prognosis in breast cancer and hepatocellular carcinoma $[7,16]$. Inhibition of Pygo2 expression increased the sensitivity of breast cancer cells to chemotherapeutic drugs and suppressed tumor growth [17]. However, the expression level of Pygo2 in GC and the relationship between Pygo2 and MDR1 was undefined. In the present study, we detected the expression level of MDR1 and Pygo2 in GC and explored the functional relationship between them.

\section{Methods}

\section{Materials}

Cis-platinum (DDP) was obtained from the first affiliated hospital of Xiamen University. MDR1 (ABCB1, Cat\# sc-55510) and $\beta$-actin (Cat\# sc-47778) antibodies were purchased from Santa Cruz Biotechnology. Pygo2 (Cat\# 11555-1-AP) antibody was obtained from Proteintech and TIAN pure Midi Plasmid Kit (Cat\# DP107) from TIANGEN. TurboFect Transfection Reagent (Cat\# R0531) was from Thermo Scientific. E.coli pLL3.7-hPygo2-KD (knock-down) and pGL3.3-control were a gift from Xiamen University School of Life Sciences.

\section{Cell culture and specimen collection}

The GC cell lines, SGC7901 and SGC7901/DDP were cultured in DMEM medium with $10 \%$ fetal bovine serum and $1 \%$ penicillin/streptomycin. The GC tissues were collected from 40 GC patients using gastroscopy and surgery. All the GC patients were treated with platinum-based neoadjuvant chemo- therapy, including combined with capecitabine, tegafur gimeracil oteracil potassium capsule (S-1), doxorubicin (ADM) and 5-fluorouracil (5-Fu). Importantly, all subjects were given written informed consent in accordance with the recommendations of Ethics and Scientific Committee of The First Affiliated Hospital of Xiamen University and the Declaration of Helsinki, the approved number from the Ethics committee of Xiamen University is 2019118.

\section{MTT assay}

Cell viability was measured by using the MTT method. Briefly, SGC7901 and SGC7901/DDP cells $\left(5 \times 10^{5} /\right.$ well $)$ were seeded in 96-well plates for $24 \mathrm{~h}$, then treated with different concentrations of DDP for $24 \mathrm{~h}$. Thereafter, cells were labeled with $20 \mu \mathrm{L}$ MTT labeling reagent, and absorbance at $570 \mathrm{~nm}$ was determined with a microplate reader.

\section{The construction of drug resistance SGC7901/DDP}

The SGC7901/DDP cell was constructed by treatment with increasing DDP concentration repeatedly and gradually. Firstly, SGC7901 cells were seeded at plates and treated with initial DDP concentration at $0.1 \mu \mathrm{g} / \mathrm{mL}$ for $24 \mathrm{~h}$, then the medium was removed, and the cells were transferred to fresh medium. The above procedures were repeated, and DDP concentration was increased gradually until the cell apoptosis rate was above $90 \%$. Cell morphology was observed by microscopy.

\section{Cell apoptosis assay}

Cell apoptosis rate was determined by flow cytometry analysis using Annexin V-FITC Apoptosis Detection Kit (KEYGEN Biotech, Cat\# KGA105KGA108). $5 \times 10^{5}$ cells were seeded in 6-well plates and treated with $0.4 \mu \mathrm{g} / \mathrm{mL}$ DDP for $24 \mathrm{~h}$. The collected cells were washed by PBS twice and then labeled with $5 \mu \mathrm{L}$ Annexin V-FITC and $5 \mu \mathrm{L}$ Propidium iodide for 5 minutes at $25{ }^{\circ} \mathrm{C}$. The stained cells were analyzed by flow cytometry using CytExpert2.0 software.

\section{Western blot assay (WB)}

Total protein extracts were prepared, and the protein concentration was measured as described previously [18]. Generally, $15 \mu \mathrm{g}$ protein was separated by $10 \%$ SDS-PAGE and transferred onto PVDF membrane, then blocked with $5 \%$ fat-free milk and incubated with primary antibodies and HRPconjugated secondary antibodies. The protein levels were detected by using the Enhanced Chemiluminescent (ECL) Detection Kit (Boster, Cat\# EK1001). 


\section{Real-time quantitative PCR}

Total RNAs were homogenized in Trizol and isolated, as described in our previous study [19]. Then cDNAs were reversely transcribed by using PrimeScript $^{\mathrm{TM}}$ RT reagent kit with gDNA Eraser (Takara, Cat\# RR047A). The expression levels of MDR1 and Pygo2 were detected using SYBR GREEN MIXTURE kit (Bio-Rad, Cat\# 10000076382) and analyzed by comparing to $\beta$-actin. The primers are listed below: Pygo2, Forward Primer: 5'-GTTTGGGC TGTCCTGAAAGTCTG-3', Reserve Primer: 5'-ATA AGGGCGCCGAAAGTTGA-3'; MDR1， Forward Primer: 5'-AGCTCGTGCCCTTGTTAGACA-3', Reserve Primer: 5'-GTCCAGGGCTTCTTGGA CAA-3'; $\beta$-actin, Forward Primer: 5'-CGAGC GGGAAATCGTGCGTGACATTAAGGAGA-3',

Reserve Primer: 5'-CGTCATACTCCTGCTTGATCCA CATCTGC-3'.

\section{Immunohistochemistry (IHC)}

GC tissues were fixed and cut into slices, then processed and incubated with primary antibodies and probed with secondary antibody. The detailed protocol could refer to our previous work [19]. Images were captured by using a Nikon microscope.

\section{pLL3.7- hPygo2-knock-down (KD) transfection}

SGC7901/DDP cells were transfected with pGL3.3-Control and pLL3.7-hPygo2-KD plasmid using TurboFect Transfection Reagent following the manufacturer's instructions. Briefly, short hairpin targeted Pygo2 (Pygo2 shRNA) and the scramble control sequences (SCR shRNA) were designed based on hPygo2 sequences and further synthesized for the construction of plasmids [20]. The detailed hPygo2 sequences could be referred to GenBank with accession number NW_925683. The primers were listed as follow: SCR shRNA, Forward Primer: 5'-GATCCCCGTGGTTTCATCGCATCTGCTTCAAG AGAGCAGATGCGATGAAACCACTTTTTA-3', Reserve Primer: 5'-AGCTTAAAAAGTGGTTTCATC GCATCTGCTCTCTTGAAGCAGATGCGATGAAAC CACGGG-3'; Pygo2 shRNA, Forward Primer: 5'GATCCCCTGTGAGGCCTCTTGTCAGAAATTCAA GAGATTTCTGACAAGAGGCCTCACATTTTTA-3', Reserve Primer: 5'-GGGACACTCCGGAGAACAGTC TTAAGTTCTCTAAAGACTGTTCTCCGGAGTGTA

AAAATTCGA-3'. The lentiviral pLL3.7 vector (X-Y Biotechnology, Cat\# XY2204) was used to express Pygo2 shRNA and pGL3.3 vector (Promega, Cat\# E1741) served as a control, the plasmids were obtained by using TIAN pure Midi Plasmid Kit and the detailed procedures were described previously [21]. The transient transfection was observed under a fluorescence microscope and verified by WB.

\section{Luciferase reporter analysis}

The pGL6-MDR1 reporter plasmid, which contained the MDR1 promoter region was constructed in our lab and described in our previous study [22]. The pGL6 reporter plasmid (318 bp) was designed as below: forward primer: 5'-CAGGGTACC AGTTGAAATGTCCCCAATGAT-3', reverse primer: 5'-CCTAGATCTGGAAAGACCTAAAGGAAACG AAC-3'. Briefly, SGC7901/DDP cells were transfected with the pGL6-MDR1 reporter plasmid, and cell homogenate was lysed and collected for luciferase reporter analysis.

\section{Chromatin immunoprecipitation (ChIP) assay}

The primers for ChIP on MDR1 were designed as described previously [22]. MDR1 primers were listed as below: forward primer: 5'-GCGTTTCTCTACTTGC CCTTTC-3', reverse primer: 5'-AGCCAATCAGCCTC ACCACAG-3'. ChIP assay was performed by using a ChIP assay kit (Merck-Millipore, Cat\# 17-371) per to the manufacturer's recommendations.

\section{Statistical analysis}

The experiments such as MTT assay, WB and RT-PCR were performed in triplicate and data was expressed as the mean \pm SD. Collective data was then analyzed using student's t-test and chi-square test. Correlation analysis was processed by using GraphPad Prism 7.0. $\quad \mathrm{P}<0.05$ was defined as the statistical significance and represented with *.

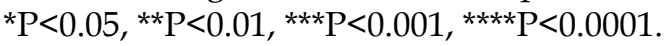

\section{Results}

\section{SGC7901/DDP was insensitive to chemotherapeutic drugs}

We successfully constructed GC drug resistance cell SGC7901/DDP after six months utilizing the method described above. Comparing to SGC7901, SGC7901/DDP had a smaller cell volume, and its cell shape had changed from long spindle to short polygon. Furthermore, SGC7901/DDP had a slower breeding rate and showed monoclonal colony growth (Fig. 1A). SGC7901/DDP was insensitive to a low concentration of DDP when cells were exposed to different concentrations of DDP (Fig. 1B). The median IC50 value of SGC7901/DDP in $24 \mathrm{~h}$ was $0.89 \mu \mathrm{g} / \mathrm{mL}$, which was significantly higher than SGC7901 with a median IC50 value in $24 \mathrm{~h}$ was $0.35 \mu \mathrm{g} / \mathrm{mL}$ (Fig. 1C). $\mathrm{ADM}$ and 5-Fu were also commonly prescribed to treat GC, we found that SGC7901/DDP was also insensitive to 5-Fu and ADM compared to SGC7901. The median IC50 values of 5-Fu and ADM in SGC7901/DDP for $24 \mathrm{~h}$ were $109.1 \mu \mathrm{g} / \mathrm{mL}$ and 1.3 $\mu \mathrm{g} / \mathrm{mL}$, which were higher than $20.5 \mu \mathrm{g} / \mathrm{mL}$ and 0.5 
$\mu \mathrm{g} / \mathrm{mL}$ in SGC7901 (Fig. 1C).

A

SGC7901

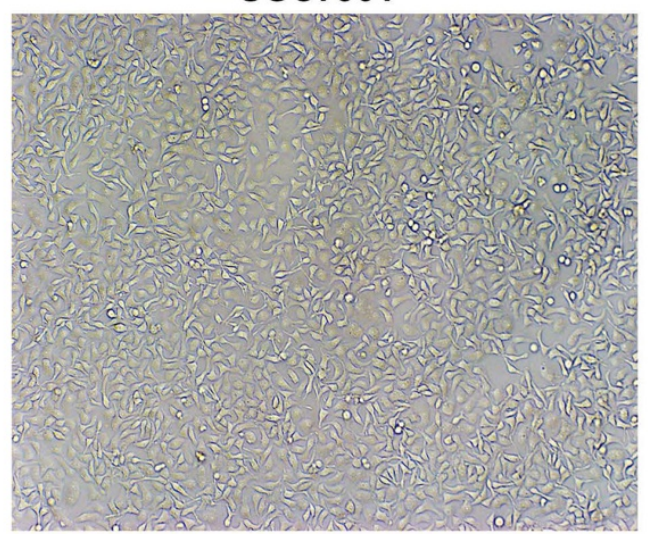

SGC7901/DDP

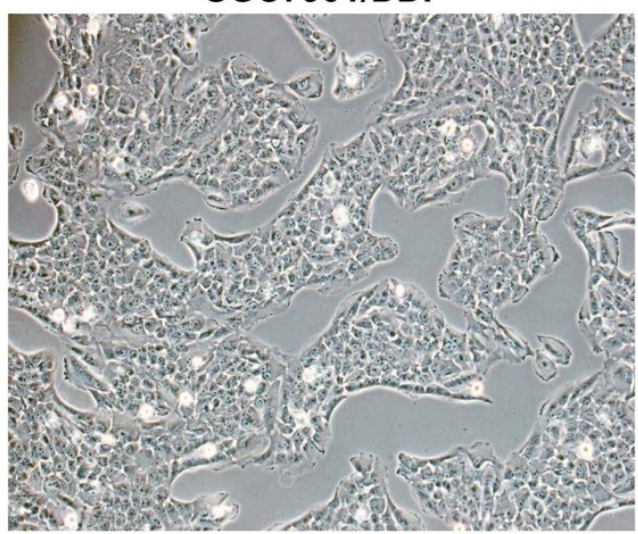

B
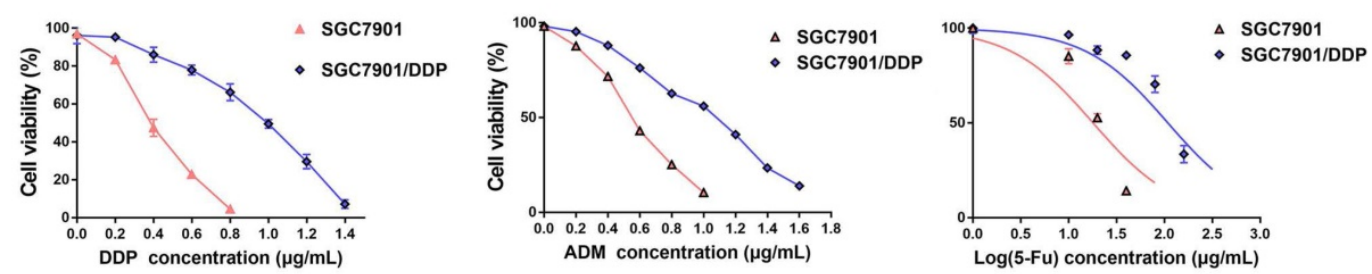

C
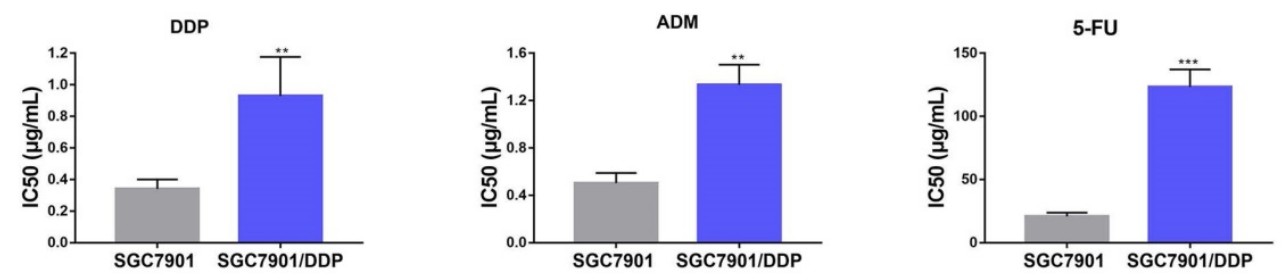

Figure 1. The construction of GC drug resistance cell SGC7901/DDP. A. SGC7901 and SGC7901/DDP was observed by using a microscopy and the images were captured, magnification: $\times 40$. B. SGC7901 and SGC7901/DDP cells were treated with different concentrations of DDP, 5-Fu and ADM for $24 \mathrm{~h}$ and the cell viability was measured by MTT assay. C. IC50 of DDP, 5-Fu and ADM in SGC7901 and SGC7901/DDP. Each experiment was repeated three times, $* * P<0.01$. ***P<0.001. DDP: cis-platinum; ADM: Adriamycin; 5-Fu: 5-fluorouracil.

Table 1. The expression status of Pygo2 and MDR1 in GC patient pre-and post-operation

\begin{tabular}{|c|c|c|c|c|}
\hline \multicolumn{2}{|l|}{ Group } & $\begin{array}{l}\text { Pre chemotherapy } \\
\text { (Gastroscopy) }\end{array}$ & $\begin{array}{l}\text { Post chemotherapy } \\
\text { (Postoperative biopsy) }\end{array}$ & \multirow{2}{*}{$\begin{array}{l}\frac{X^{2} \text {-test }}{P \text { value }} \\
<0.0001\end{array}$} \\
\hline Pygo2 & Overexpression & $6(15 \%)$ & $25(62.5 \%)$ & \\
\hline & $\begin{array}{l}\text { None } \\
\text { overexpression }\end{array}$ & $34(85 \%)$ & $15(37.5 \%)$ & \\
\hline \multirow[t]{2}{*}{ MDR1 } & Overexpression & $2(5 \%)$ & $18(45 \%)$ & $<0.0001$ \\
\hline & $\begin{array}{l}\text { None } \\
\text { overexpression }\end{array}$ & $38(95 \%)$ & $22(55 \%)$ & \\
\hline
\end{tabular}

\section{High expression of Pygo2 and MDR1 in SGC7901/DDP and GC patients after chemotherapy}

Firstly, we detected the protein and mRNA expression levels of Pygo2 and MDR1 in SGC7901/DDP by WB and RT-PCR. Our results showed that SGC7901/DDP had higher expression levels of Pygo2 and MDR1 compared with SGC7901 (Fig. 2A and 3A). Next, we analyzed Pygo2 and
MDR1 expression levels in normal-appearing tissue adjacent to GC (NaT), GC tissue (GcT) and GC tissue after neoadjuvant chemotherapy $(\mathrm{NeC})$. Our data revealed that MDR1 and Pygo2 was low or rarely expressed in $\mathrm{NaT}$ but were highly expressed in GcT and with highest expression levels in $\mathrm{NeC}$ (Fig. 2A, Fig. 2B and 3B).

\section{Pygo2 overexpression preceded MDR1 and Pygo2 correlated with MDR 1}

We analyzed 40 GC patients in the first affiliated hospital of Xiamen University who had undergone neoadjuvant DDP-based chemotherapy. Our results showed that $15 \%(6 / 40)$ of GC patients were Pygo2 overexpression positive, and 5\% (2/40) were MDR1 overexpression positive pre-chemotherapy. While amongst the group, $62.5 \%(25 / 40)$ of GC patients were Pygo2 overexpression positive, and $45 \%(18 / 40)$ were MDR1 overexpression positive post-chemotherapy. The results indicated that Pygo2 overexpression 
A

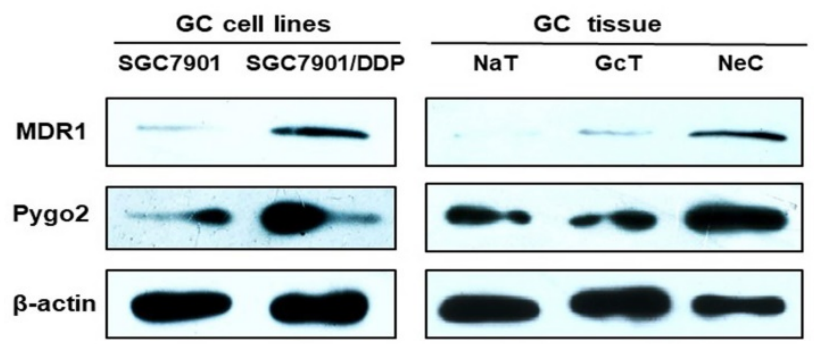

B

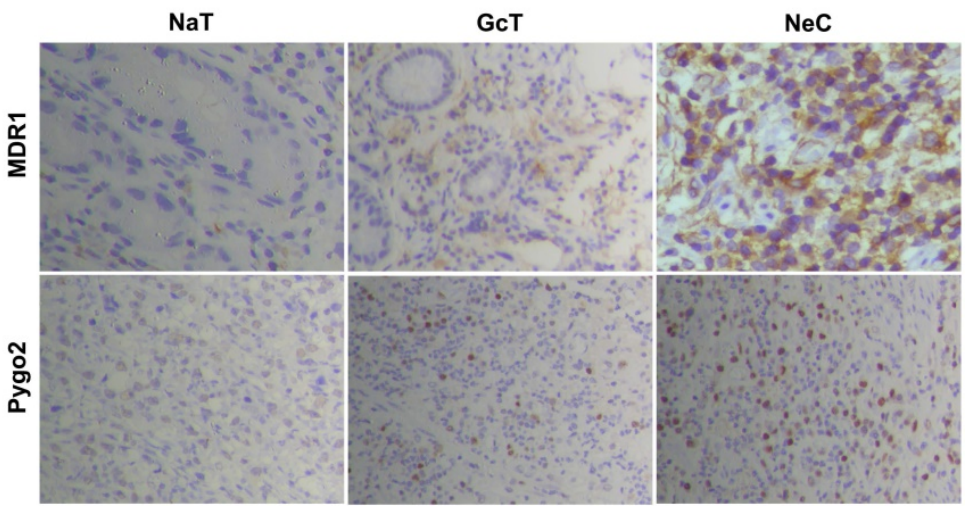

Figure 2. Pygo2 and MDR1 were overexpression in GC cell lines and GC patients after chemotherapy. A.The Pygo2 and MDR1 protein expression levels were detected by WB. B. The expression levels of Pygo2 and MDR1 in GC tissue measured by IHC, magnification: $\times 40$. IHC: immunohistochemistry; $\mathrm{NaT}$ : normal-appearing tissue adjacent to GC, GcT: GC tissue; $\mathrm{NeC}$ : GC tissue after neoadjuvant chemotherapy.

might precede MDR1 (Table 1). Furthermore, analyzing the two genes across online data sets showed that a strong correlation between Pygo2 and MDR1 expression levels after chemotherapy in GC tissue (Fig. 3C).

\section{The knock-down of Pygo2 induced MDR I downregulation and rendered GC cell sensitive to chemotherapeutic drugs}

To clarify the relationship between Pygo2 and MDR1 in GC, we suppressed Pygo2 expression in SGC7901/DDP by transfecting pLL3.7-hPygo2-KD plasmid. We proceeded to perform WB to determine whether Pygo2 was successfully suppressed (Fig. 4A). The results suggested that when Pygo2 was inhibited, MDR1 expression was also downregulated (Fig. 4B). In addition, GC drug resistance cell SGC7901/DDP became sensitive to chemotherapeutic drugs when Pygo2 was knocked down (Fig. 4C).

\section{Pygo2 promoted MDR1 activation by interacting with MDR 1}

To illuminate the role of Pygo2 in the activation of MDR1, we performed luciferase reporter analysis. We found that Pygo2 could promote the activation of MDR1 promoter (Fig. 4D). Further mechanism analysis by ChIP assay verified the association of Pygo2 with the MDR1 promoter region (Fig. 4E). One other point worth emphasizing is our previous study also demonstrated Pygo2 could modulate MDR1 activation in breast cancer by directly binding to $\beta$-catenin, thus regulating the $W n t / \beta$-catenin signaling pathway [7].

A
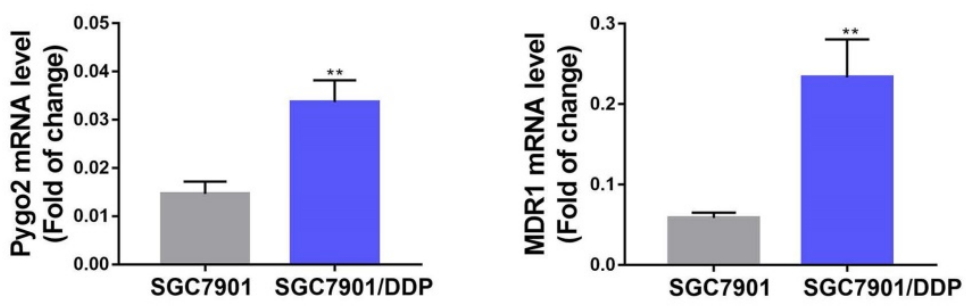

B

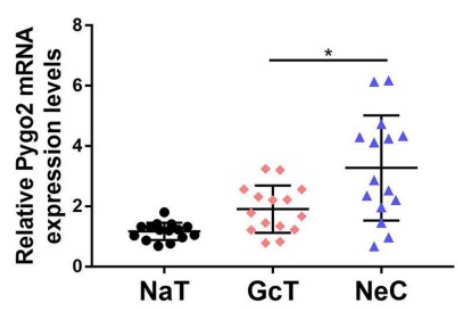

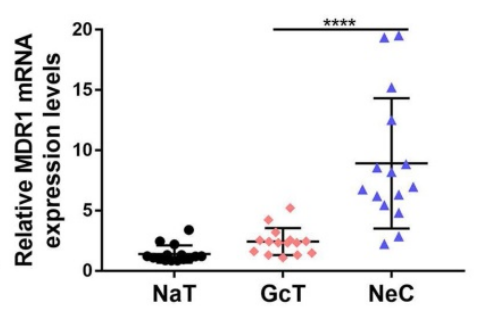

C

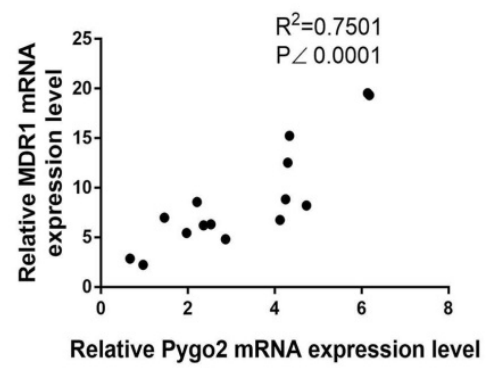

Figure 3. Pygo2 overexpression was strong corelated with MDR1. The Pygo2 and MDR1 RNA expression levels were detected by RT-PCR in GC cell lines (A) and GC tissues (B). C.The Pearson's R correlation was analyzed between Pygo2 and MDR1 expression levels in GC patients after chemotherapy. NaT: normal-appearing tissue adjacent to GC, GcT: GC tissue; NeC: GC tissue after neoadjuvant chemotherapy. 
A

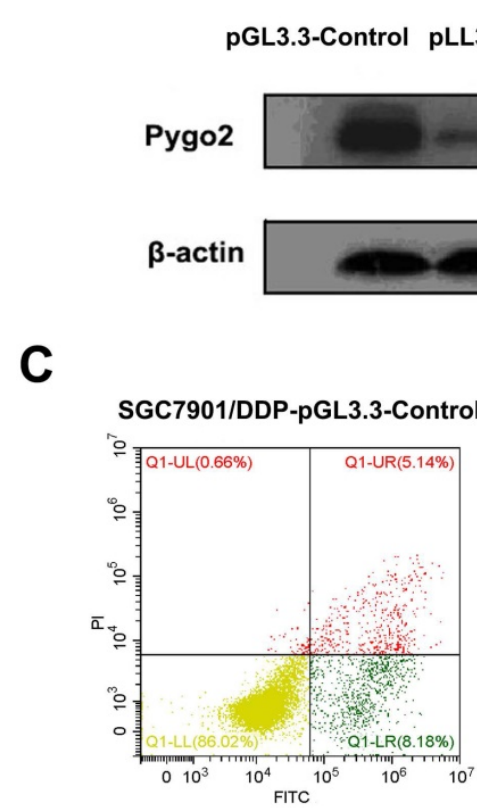

D

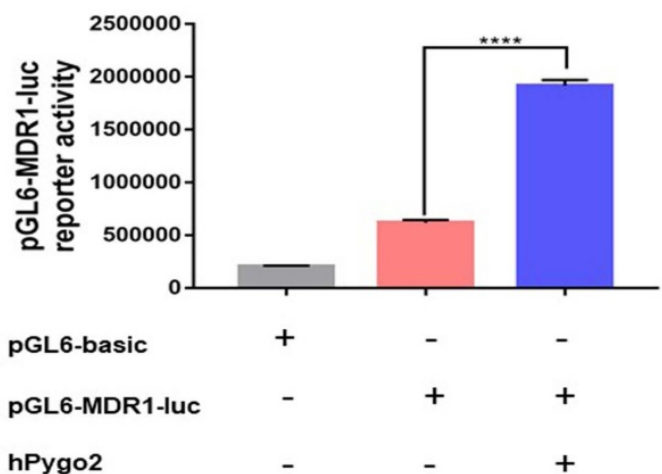

B

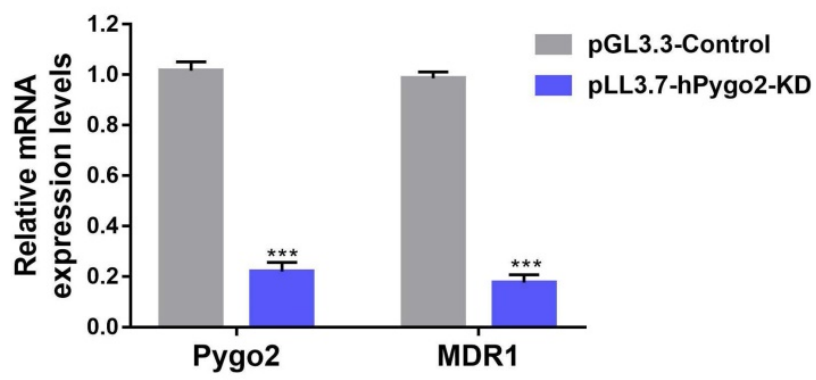

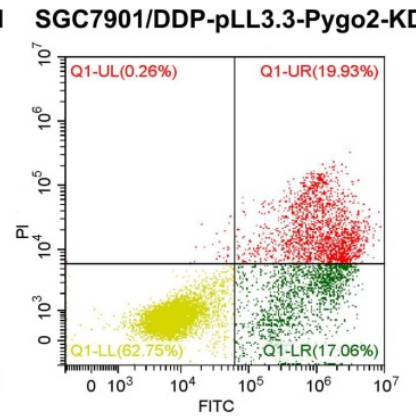

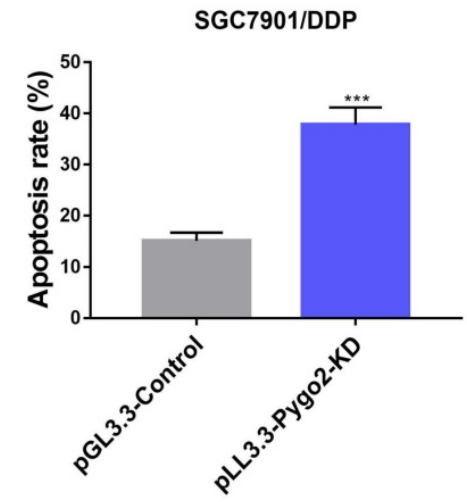

$\mathbf{E}$

Figure 4. Pygo2 modulated MDR1 expression in GC. A. The knock-down of Pygo2 down was identified by WB after transfection. B. The expression levels of Pygo2 and MDR1 in SGC7901/DDP-pLL3.3-Pygo2-KD and SGC7901/DDP-pGL3.3. C. SGC7901/DDP-pLL3.3-Pygo2-KD and SGC7901/DDP-pGL3.3 were treated with $0.4 \mu \mathrm{gg} / \mathrm{mL} \mathrm{DDP} \mathrm{for} 24 \mathrm{~h}$, the apoptosis rate was analyzed by flow cytometry. D. SGC7901/DDP cells were transfected with the indicated plasmids and MDR 1-reporter gene luciferase activity was measured. E. The interaction between Pygo2 and MDR1 promoter region using anti-Pygo2 antibody in SGC7901/DDP and GC tissue after neoadjuvant chemotherapy (Nec) by ChIP assay. $* * * P<0.001$

Taking into consideration the obtained results; we can conclude that the overexpression of Pygo2 promotes MDR1 activation and is involved in DDP drug resistance in GC.

\section{Discussion}

Majority of GC patients are diagnosed at advanced stage disease as GC has a relatively low early detection and diagnosis rate. Chemotherapy is an important alternative therapeutic method for these patients. However, the development of MDR not only causes failure of the first-line treatment recommended by National Comprehensive Cancer Network (NCCN), but also results in the ineffectiveness of the second-and third-line solutions [23]. Therefore, it is important to elucidate the mechanism of chemoresistance.

Studies exploring the mechanism of drug resistance are mainly divided into the following categories: alterations in drug efflux [24], dysfunction of DNA damage repair [25], disruption of apoptosis balance such as overexpression of anti-apoptosis proteins [26] and mutation of drug targets [27]. One of the most studied mechanism is overexpression of MDR1 and multi-drug resistant associate protein (MRP), which serve as drug efflux transporters leading to a decrease in intracellular drug concentration [28]. MDR1 overexpression was 
identified in GC cell lines and primary GC tissue in previous studies [29]. Additionally, MDR1 overexpression was negatively associated with chemosensitivity [30]. Studies on MDR transporter inhibitors have been flourishing, but with little success [31]. Hence, it was essential to identify novel MDR biomarkers and drug targets.

Pygo2 is a novel effector protein, downstream of the Wnt/ $\beta$-catenin signaling pathway, that acts as a co-activator with $\beta$-catenin to promote $\beta$-catenin/LEF/TCF activation [32]. Abnormal expression of Pygo2 has been reported in several cancers such as breast cancer, glioma, esophageal squamous cell carcinoma, hepatocellular carcinoma and prostate cancer [7, 13, 14, 16, 33-35]. Furthermore, Pygo2 overexpression was associated with tumor initiation and progression, malignant phenotype, and poor prognosis [13, 15, 16, 34]. Inhibition of Pygo2 suppressed tumor growth, invasion, and epithelialmesenchymal transition (EMT) [17]. Our previous study showed that Pygo2 was associated with chemoresistance in breast cancer by activating MDR1 [7]. MDR1 was the downstream target of the Wnt/ $\beta$ catenin signaling pathway, and it was upregulated when the Wnt $/ \beta$-catenin was activated [36]. Pygo2 not only promoted $\mathrm{Wnt} / \beta$-catenin activation but also modulated Wnt/ $\beta$-catenin activity in tissue-and gene-dependent manner $[11,37,38]$. Thus, whether Pygo2 regulates MDR1 in GC or not still needs further study.

These recent findings verified that Pygo2 and MDR1 were overexpressed in GC, especially in GC patients after chemotherapy. Pygo2 overexpression precedes MDR1 and Pygo2 strongly correlated with MDR1 in GC patients who underwent neoadjuvant chemotherapy. The Knock-down of Pygo2 could restore the drug-resistant cell SGC/7901 sensitivity to chemotherapeutic drugs. Further mechanism analysis indicated that Pygo2 could promote MDR1 activation by binding to the MDR1 promoter region. These results demonstrated that Pygo2 might be a novel biomarker for monitoring drug resistance by upregulating MDR1. This study also provided a potential therapeutic target in the treatment of cancers where Pygo2 is overexpressed.

In conclusion, Pygo2 and MDR1 were overexpressed in GC patients after chemotherapy. Pygo2 played a pivotal role in drug resistance in GC by promoting MDR1 activation. Pygo2 may be a novel biomarker for monitoring drug resistance and a potential therapeutic target for cancer treatment in the future.

\section{Acknowledgements}

We appreciate the support from Professor Bo-an
$\mathrm{Li}$ for designing the pLL3.7-hPygo2-KD and pGL3.3-control at the Department of Biomedical Sciences, Xiamen University School of Life Sciences, Xiang' an Campus of Xiamen University.

\section{Funding}

This work was supported by the Natural Science Foundation of Fujian program (grant number 2019J01575) and the Hubei Education Program (grant number JX5B78).

\section{Authors' contributions}

MQC and YHD provided the direction of study. DDZ performed the major experiments, analyzed the data and wrote the manuscript, QWW guided the experiments. YHZ contributed to the specimen collection and contributed to the cell experiments. YL helped analyzing the ICH. NK and ZMZ helped revise the manuscript. All authors read and approved the final manuscript.

\section{Competing Interests}

The authors have declared that no competing interest exists.

\section{References}

1. Choi $\mathrm{YH}, \mathrm{Yu}$ AM. $\mathrm{ABC}$ transporters in multidrug resistance and pharmacokinetics, and strategies for drug development. Current pharmaceutical design. 2014; 20: 793-807.

2. Robey RW, Pluchino KM, Hall MD, Fojo AT, Bates SE, Gottesman MM. Revisiting the role of $\mathrm{ABC}$ transporters in multidrug-resistant cancer. Nature reviews Cancer. 2018; 18: 452-64.

3. Wolking S, Schaeffeler E, Lerche H, Schwab M, Nies AT. Impact of Genetic Polymorphisms of ABCB1 (MDR1, P-Glycoprotein) on Drug Disposition and Potential Clinical Implications: Update of the Literature. Clinical pharmacokinetics. 2015; 54: 709-35.

4. Ceballos MP, Rigalli JP, Ceré LI, Semeniuk M, Catania VA, Ruiz ML. ABC Transporters: Regulation and Association with Multidrug Resistance in Hepatocellular Carcinoma and Colorectal Carcinoma. Current medicinal chemistry. 2019; 26: 1224-50.

5. Adamska A, Falasca M. ATP-binding cassette transporters in progression and clinical outcome of pancreatic cancer: What is the way forward? World journal of gastroenterology. 2018; 24: 3222-38.

6. Levi M, Brunetti B, Sarli G, Benazzi C. Immunohistochemical Expression of P-glycoprotein and Breast Cancer Resistance Protein in Canine Mammary Hyperplasia, Neoplasia and Supporting Stroma. Journal of comparative pathology. 2016; 155: 277-85.

7. Zhang ZM, Wu JF, Luo QC, Liu QF, Wu QW, Ye GD, et al. Pygo2 activates MDR1 expression and mediates chemoresistance in breast cancer via the Wnt/ $\beta$-catenin pathway. Oncogene. 2016; 35: 4787-97.

8. Thompson B, Townsley F, Rosin-Arbesfeld R, Musisi H, Bienz M. A new nuclear component of the Wnt signalling pathway. Nature cell biology. 2002; 4: 367-73.

9. Kessler R, Hausmann G, Basler K. The PHD domain is required to link Drosophila Pygopus to Legless/beta-catenin and not to histone H3. Mechanisms of development. 2009; 126: 752-9.

10. Chen J, Luo Q, Yuan Y, Huang X, Cai W, Li C, et al. Pygo2 associates with MLL2 histone methyltransferase and GCN5 histone acetyltransferase complexes to augment Wnt target gene expression and breast cancer stem-like cell expansion. Molecular and cellular biology. 2010; 30: 5621-35.

11. Gu B, Sun P, Yuan Y, Moraes RC, Li A, Teng A, et al. Pygo2 expands mammary progenitor cells by facilitating histone $\mathrm{H} 3 \mathrm{~K} 4$ methylation. The Journal of cell biology. 2009; 185: 811-26.

12. Schwab KR, Patterson LT, Hartman HA, Song N, Lang RA, Lin X, et al. Pygo1 and Pygo2 roles in Wnt signaling in mammalian kidney development. BMC biology. 2007; 5: 15 .

13. Lu X, Pan X, Wu CJ, Zhao D, Feng S, Zang Y, et al. An In vivo Screen Identifies PYGO2 as a Driver for Metastatic Prostate Cancer. Cancer research. 2018; 78: 3823-33.

14. Moghbeli M, Sadrizadeh A, Forghanifard MM, Mozaffari HM, Golmakani E, Abbaszadegan MR. Role of Msi1 and PYGO2 in esophageal squamous cell 
carcinoma depth of invasion. Journal of cell communication and signaling. 2016; 10: 49-53.

15. Liu Y, Dong QZ, Wang S, Fang CQ, Miao Y, Wang L, et al. Abnormal expression of Pygopus 2 correlates with a malignant phenotype in human lung cancer. BMC cancer. 2013; 13: 346.

16. Zhang S, Li J, He F, Wang XM. Abnormal nuclear expression of Pygopus-2 in human primary hepatocellular carcinoma correlates with a poor prognosis. Histopathology. 2015; 67: 176-84.

17. Chi Y, Wang F, Zhang T, Xu H, Zhang Y, Shan Z, et al. miR-516a-3p inhibits breast cancer cell growth and EMT by blocking the Pygo2/Wnt signalling pathway. Journal of cellular and molecular medicine. 2019; 23: 6295-307.

18. Zhang D, Liu Y, Luo Z, Chen Y, Xu A, Liang Y, et al. The novel thioredoxin reductase inhibitor A-Z2 triggers intrinsic apoptosis and shows efficacy in the treatment of acute myeloid leukemia. Free radical biology \& medicine. 2020; 146: $275-86$.

19. Dongdong Z, Jin Y, Yang T, Yang Q, Wu B, Chen Y, et al. Antiproliferative and Immunoregulatory Effects of Azelaic Acid Against Acute Myeloid Leukemia via the Activation of Notch Signaling Pathway. Frontiers in pharmacology. 2019; 10: 1396.

20. Wang ZX, Chen YY, Li BA, Tan GW, Liu XY, Shen SH, et al. Decreased pygopus 2 expression suppresses glioblastoma U251 cell growth. Journal of neuro-oncology. 2010; 100: 31-41.

21. Xie YY, Mo CL, Cai YH, Wang WJ, Hong XX, Zhang KK, et al. Pygo2 Regulates Adiposity and Glucose Homeostasis via $\beta$-Catenin-Axin2-GSK3 $\beta$ Signaling Pathway. Diabetes. 2018; 67: 2569-84.

22. Chen S, Wang H, Li Z, You J, Wu QW, Zhao C, et al. Interaction of WBP2 with ERa increases doxorubicin resistance of breast cancer cells by modulating MDR1 transcription. British journal of cancer. 2018; 119: 182-92.

23. Thiels CA, Hanson KT, Habermann EB, Boughey JC, Grotz TE. Integrated cancer networks improve compliance with national guidelines and outcomes for resectable gastric cancer. Cancer. 2020; 126: 1283-94.

24. Cui H, Zhang AJ, Chen M, Liu JJ. ABC Transporter Inhibitors in Reversing Multidrug Resistance to Chemotherapy. Current drug targets. 2015; 16: 1356-71.

25. Özeş AR, Miller DF, Özeș ON, Fang F, Liu Y, Matei D, et al. NF-kB-HOTAIR axis links DNA damage response, chemoresistance and cellular senescence in ovarian cancer. Oncogene. 2016; 35: 5350-61.

26. Xia H, Hui KM. Mechanism of cancer drug resistance and the involvement of noncoding RNAs. Current medicinal chemistry. 2014; 21: 3029-41.

27. Zugazagoitia J, Guedes C, Ponce S, Ferrer I, Molina-Pinelo S, Paz-Ares L. Current Challenges in Cancer Treatment. Clinical therapeutics. 2016; 38: 1551-66.

28. Cole SP. Multidrug resistance protein 1 (MRP1, ABCC1), a "multitasking" ATP-binding cassette $(\mathrm{ABC})$ transporter. The Journal of biological chemistry. 2014; 289: 30880-8.

29. MDR1 gene expression and its clinical relevance in primary gastric carcinomas. Cancer. 1993; 71: 667-71.

30. Li Y, Tan BB, Zhao Q, Fan LQ, Liu Y, Wang D. Regulatory mechanism of ZNF139 in multi-drug resistance of gastric cancer cells. Molecular biology reports. 2014; 41: 3603-10.

31. Li W, Zhang $\mathrm{H}$, Assaraf YG, Zhao $\mathrm{K}, \mathrm{Xu} \mathrm{X,} \mathrm{Xie} \mathrm{J,} \mathrm{et} \mathrm{al.} \mathrm{Overcoming} \mathrm{ABC}$ transporter-mediated multidrug resistance: Molecular mechanisms and novel therapeutic drug strategies. Drug resistance updates: reviews and commentaries in antimicrobial and anticancer chemotherapy. 2016; 27: 14-29.

32. Townsley FM, Cliffe A, Bienz M. Pygopus and Legless target Armadillo/betacatenin to the nucleus to enable its transcriptional co-activator function. Nature cell biology. 2004; 6: 626-33.

33. Moghbeli M, Abbaszadegan MR, Farshchian M, Montazer M, Raeisossadati R, Abdollahi A, et al. Association of PYGO2 and EGFR in esophageal squamous cell carcinoma. Medical oncology (Northwood, London, England). 2013; 30: 516.

34. Watanabe K, Fallahi M, Dai X. Chromatin effector Pygo2 regulates mammary tumor initiation and heterogeneity in MMTV-Wnt1 mice. Oncogene. 2014; 33: 632-42.

35. Li M, Chao L, Wu J, Xu H, Shen S, Chen S, et al. Pygo2 siRNA Inhibit the Growth and Increase Apoptosis of U251 Cell by Suppressing Histone H3K4 Trimethylation. Journal of molecular neuroscience : MN. 2015; 56: 949-55.

36. Flahaut M, Meier R, Coulon A, Nardou KA, Niggli FK, Martinet D, et al. The Wnt receptor FZD1 mediates chemoresistance in neuroblastoma through activation of the Wnt/beta-catenin pathway. Oncogene. 2009; 28: 2245-56.

37. Sun $\mathrm{P}$, Watanabe $\mathrm{K}$, Fallahi M, Lee B, Afetian ME, Rheaume C, et al. Pygo2 regulates $\beta$-catenin-induced activation of hair follicle stem/progenitor cells and skin hyperplasia. Proceedings of the National Academy of Sciences of the United States of America. 2014; 111: 10215-20.

38. Gu B, Watanabe K, Sun P, Fallahi M, Dai X. Chromatin effector Pygo2 mediates Wnt-notch crosstalk to suppress luminal/alveolar potential of mammary stem and basal cells. Cell stem cell. 2013; 13: 48-61. 